\title{
A REVIEW ON THE STABILITY OF LIQUID BRIDGES
}

\author{
J. Meseguer,* L. A. Slobozhanin** and J. M. Perales* \\ * LamfETSIA, Laboratorio de Aerodinámica, E.T.S.I. Aeronónticos, \\ Universidad Politécnica de Madrid, 28040 Madrid, Spain \\ ** Institute of Low Temperature Physics and Engineering. Academy of \\ Sciences of Ukraine, 47 Lenin Avenue, 310164 Kharkov, Ukraine
}

\begin{abstract}
Errors in theAlthough early studies dealing with the stability of liquid bridges were published long time ago, these studies were mainly concerned with the stability of axisymmetric liquid bridges between parallel, coaxial, equal-in-diameter solid disks, with regard to axisymmetric perturbations. Results including effects such as solid rotation of the liquid column, supporting disks of different diameters and an axial acceleration acting parallel to the liquid column can be found in several works published in the early eighties, although most of these analysis were restricted to liquid bridge configurations having a volume of liquid equal or close enough to that of a cylinder of the same radius. Leaving apart some asymptotic studies, the analysis of non-axisymmetric effects on the stability of liquid bridges (lateral acceleration, eccentricity of the supporting disks) and other not so-classical effects (electric field) has been initiated much more recently, the results concerning these aspect of liquid bridge stability being yet scarce.
\end{abstract}

\section{INTRODUCTION}

The liquid bridge is a microgravity relevant configuration for its own merit as a simple and controllable setup for basic fluid science studies and for its direct application to the crystal growth technique known as floating zone. In the simplest configuration, a liquid bridge consists of an isothermal mass of liquid held by surface tension forces between two parallel solid disks, as sketched in Figure 1. Such fluid configuration can be uniquely defined by the following set of dimensionless parameters: the slenderness, $A=$ $L /\left(2 R_{0}\right)$, where $L$ is the distance between the supporting disks and $R_{0}=\left(R_{1}+R_{2}\right) / 2$ the mean radius, which is used as characteristic length; the ratio of the radius of the smaller disk, $R_{1}$, to the radius of the larger one, $R_{2}$, that is $K=R_{1} / R_{2}$ (although in some cases the equivalent parameter $h=(1-K) /(1+K)$ has been used instead of $K)$; the dimensionless eccentricity, $e=E / R_{0}, 2 E$ being the distance between the disk axes; the dimensionless volume of liquid, $V=\bar{V} /\left(\pi R_{0}^{2} L\right)$, where $\bar{V}$ stands for the physical volume; the axial Bond number, $B_{a}=\rho g_{a} R_{0}^{2} / \sigma$, where $\rho$ is the difference between the liquid bridge density and the surrounding medium density (either a gas or another liquid), $g_{a}$ the axial acceleration and $\sigma$ the surface tension, the lateral Bond number

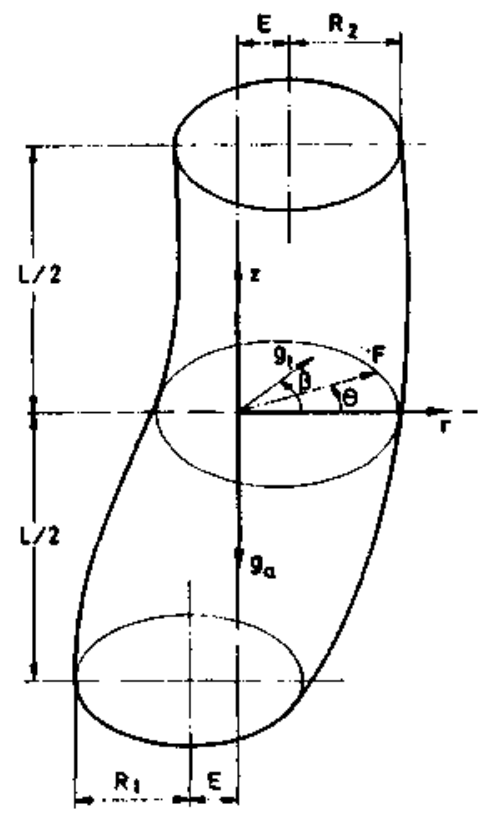

Fig. 1. Geometry and coordinate system for the liquid bridge problem. 
$B_{l}=\rho g_{l} R_{0}^{2} / \sigma$, where $g_{l}$ stands for the lateral component of acceleration (which forms an angle $\beta$ with respect to the plane defined by the axes of the disks) and, if it is assumed that the liquid bridge is rotating as a solid body with angular velocity $\omega$, the Weber number, $W e=\rho \omega^{2} R_{0}^{3} / \sigma$.

Because of the large number of parameters involved $\left(A, V, K, B_{a}, W e, e, B_{l}\right)$ the study of the equilibrium shapes and stability limits of liquid bridges is a cumbersome task which has focussed the attention of many investigators during decades. Up to now most of the published results deal with initially axisymmetric configurations $\left(e=B_{l}=0\right)$ and only recently some results conceming non-axisymmetric liquid bridges have become available. Of course the above mentioned set of parameters does not define entirely an arbitrary liquid bridge configuration; one can easily imagine liquid bridges between non-circular or even non-planar supports, etc..., but these effects, to our knowledge, have not been considered but marginally in the literature.

From the mathematical point of view, equilibrium shapes of liquid bridges are described by the Young-Laplace equation, which in dimensionless variables reads

$$
M(F)+P-B_{a} z+B_{l} F \cos (\theta-\beta)+\frac{1}{2} F^{2} W e=0,
$$

where $M(F)$ is twice the mean curvature of the interface, that is

$$
\begin{aligned}
M(F)= & \left\{F\left[1+\left(F_{2}\right)^{2}\right]\left[F_{\theta \theta}-F\right]+F F_{22}\left[F^{2}+\left(F_{\theta}\right)^{2}\right]-2 F_{\theta}\left[F_{\theta}+F F_{z} F_{2 \theta}\right]\right\} \\
& \left\{F^{2}\left[1+\left(F_{1}\right)^{2}\right]+\left(F_{\theta}\right)^{2}\right\}^{-3 / 2}
\end{aligned}
$$

Boundary conditions are

$$
\begin{aligned}
& F( \pm \Lambda, \theta)=\left[(1 \pm h)^{2}-e^{2} \sin ^{2} \theta\right]^{1 / 2} \pm e \cos \theta, \\
& F(z, \theta+2 \pi)=F(z, \theta), \\
& \frac{1}{2} \int_{-\Lambda}^{\Lambda} d z \int_{0}^{2 \pi} F^{2} d \theta=2 \pi \Lambda V .
\end{aligned}
$$

To write down the above expressions all lengths have been made dimensionless with $R_{0} ; F=F(z, \theta)$ stands for the shape of the interface, and the parameters appearing in the problem formulation, already introduced, are the slenderness, $A$, the dimensionless volume, $V$, the dimensionless eccentricity, $e$, the dimensionless disk radii difference, $h$, and the two components of Bond number, $B_{a}$ and $B_{l}$, respectively. $P$ is a constant (related with the difference between the outer pressure and the inner pressure), which has been made dimensionless with $\sigma / R_{0}$. The subscripts $z$ and $\theta$ indicate derivatives with respect to $z$ and $\theta$, respectively.

In the following, stability diagrams in the $A-V$ plane of difterent liquid bridge configurations are presented. First of all the stability diagram of liquid bridges between coaxial, equal disks, in gravitationless conditions and without considering any solid body rotation $\left(e=h=B_{a}=B_{l}=\right.$ $W e=0$ ), is presented, such fluid configuration being denoted as basic configuration. In the following sections the influence of axisymmetric effects like non-zero axial Bond number, $B_{a} \neq 0$, and/or unequal disks, $h \neq 0$, are considered and, finally, solid body rotation and 
non-axisymmetric effects such as eccentricity, $e \neq 0$, and lateral Bond number, $B_{l} \neq 0$, are taken into account.

\section{BASIC CONFIGURATIONS}

The stability diagram for axisymmetric liquid bridges with $e=h=B_{a}=B_{l}=W e=0$ is shown in Figure 2. Early stability studies conceming basic configurations were published more than 20 year ago by Gillette and Dyson /1/. The most relevant results concerning the stability of such

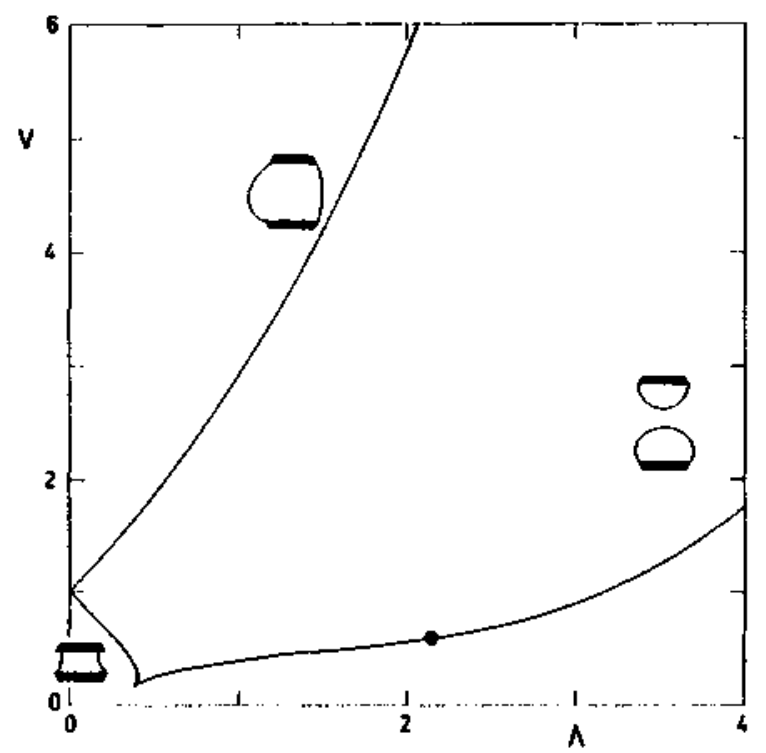

Fig. 2. Stability diagram of axisymmetric liquid bridges between coaxial, equal disks under gravitationless conditions without solid body rotation $\left(e=h=B_{a}=B_{l}=W e=0\right)$. configurations were published in the decade of the seventies and early eighties, both from the theoretical $/ 2$, $3,4,5,6 /$ and experimental $/ 7,8,9 /$ points of view. The maximum volume of an axisymmetric liquid bridge is determined by the appearance of a non-axisymmetric perturbation which causes a non-axisymmetric deformation of the liquid bridge interface and a (possibly not reversible) spreading of the liquid over the lateral surfaces of the supporting disks, whereas the smaller possible value of the volume (the minimum volume stability limit) is driven by an axisymmetric perturbation whose nature depends on the slenderness. For large slendernesses, $A>2.13$, the minimum volume stability limit is determined by the appearance of a catastrophic pitch-fork bifurcation to non-symmetric (with respect to the middle plane parallel to the disks)

unstable equilibrium shapes (indeed the corresponding eigenfunction is antisymmetric with respect to the middle plane). On the other hand, for small slendernesses, $A<2.13$, the stability limit is determined by a turning point in the bifurcation diagram and the associate unstable equilibrium shapes are still symmetric with respect to the middle plane parallel to the disks. This means that when the stability limit is reached and the breakage of the liquid column takes place, the volume of each one of the two main drops appearing after the breaking will be equal if $A<2.13$, but the two drops will be of different volume if $A>2.13$. If the slenderness is small enough there is another constraint fixing the minimum volume of the liquid column: a non-axisymmetric instability appears and, depending on the contact angle, a possible detachment of the liquid bridge interface from the edges of the disks could happen.

\section{UNEQUAL DISKS AND AXIAL BOND NUMBER}

The influence on stability limits of having disks with different diameters was mainly investigated in the last decade $/ 10,11 /$. The available results concern mainly the stability limits of minimum volume. The influence of unequal disks in the $\Lambda-V$ stability diagram is shown in Figure 3. As it can be observed the stable region in the $A-V$ plane decreases as the parameter $h$ increases ( $K$ decreases). The problem of the calculation of the maximum volume of liquid bridges between unequal disks has been very recently solved and the results are still unpublished $/ 12 /$. 


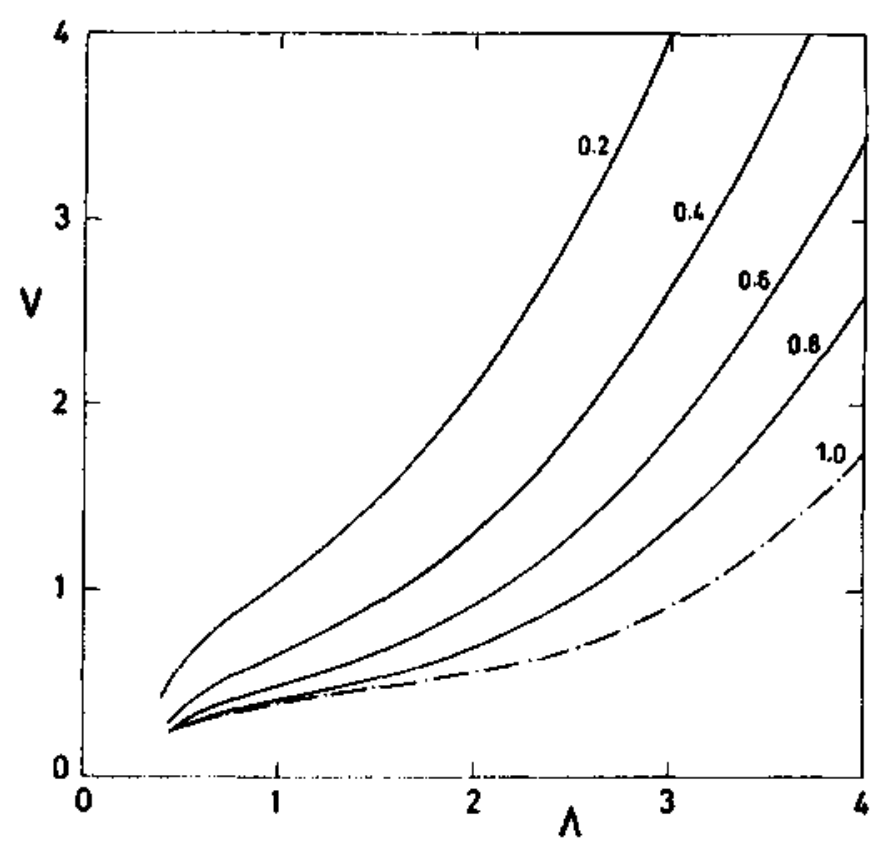

Fig. 3. Stability diagram of axisymmetric liquid bridges between coaxial, unequal disks under gravitationless conditions without solid body rotation $\left(e=B_{a}=B_{l}=W e=0\right.$, $K \neq 1$ ). Numbers on the curves indicate the value of the parameter $K$.

Concerning the influence of axial Bond number, early studies $/ 13,14,15 /$ were concemed with the maximum stable slenderness of liquid bridges between equal disks having cylindrical volume $(V=1)$. Different attempts have been made to calculate the minimum volume stability limit as well as the maximum one both from the theoretical $/ 16,17 /$ and experimental point of view $/ 17,18 /$. This problem has been extensively analyzed in a work which has been recently published $/ 19 /$ and the results are summarized in Figure 4 . As can be seen, for any non zero value of the axial Bond number the stability limit can be represented by a single closed curve in the $A-V$ plane. In such a stability limit curve it is possible to distinguish, as in the previous cases, three different parts. For very small volumes and very small slenderness the instability is governed by the detachment of the interface from the edge of the top disk. Another part of the stability limit is characterized by the axisymmetric breakage of the liquid column (that part corresponds to the minimum volume) whereas the last part of the curve is characterized by the loss of axisymmetry of the equilibrium shapes and the appearance of non-axisymmetric deformations of the interface (maximum volume stability limit).

The combined influence of both unequal disks and axial Bond number on stability limits of minimum volume was first studied through an asymptotic analysis by the mid eighties $/ 20 \%$. If the effect of axial gravity and the effect of unequal disks are considered separately, each one of them reduce the stable region, as can be expected. Nevertheless, since both effects are non-symmetric with respect to the middle plane parallel to the disks, under certain circumstances (when they are in opposition) they can compensate to a certain extent (this happens when axial gravity is directed towards the smaller disk). The more complete results up to now published concerning the combined influence of both $h \neq 0$ and $B_{a} \neq 0$ can be found in Perales, Meseguer and Martínez $/ 21$, where the minimum volume stability limits are analyzed both theoretically and experimentally. A typical set of curves of minimum volume stability limits is shown in Figure 5. As it can be observed, when both disks are not of the same diameter, the stability limit corresponding to a positive value of axial Bond number provides a stable region larger than that corresponding to $B_{a}=0$, showing the stabilizing effect of axial Bond number in this case. 


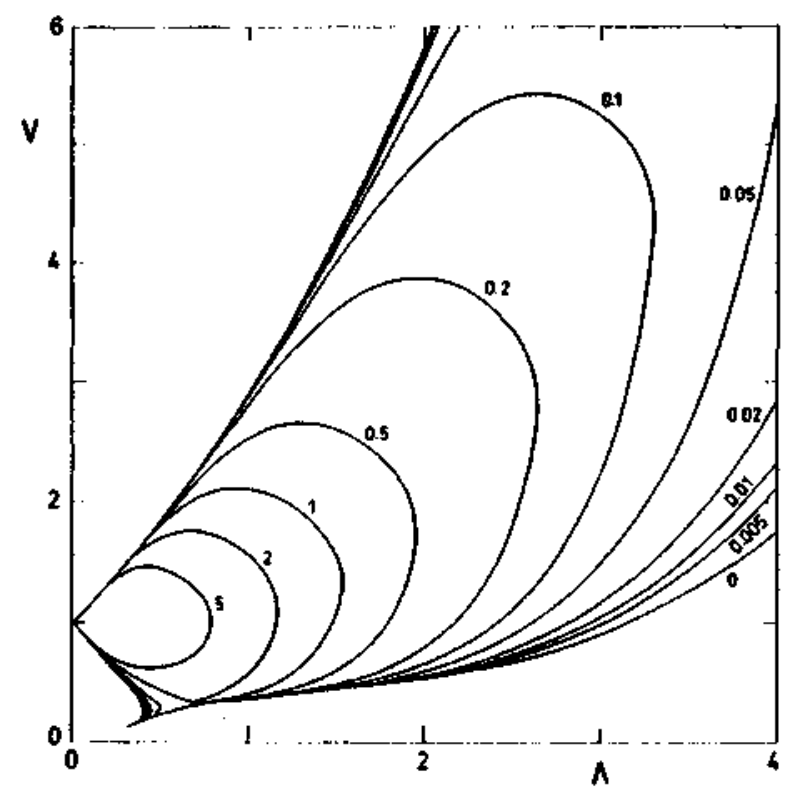

Fig. 4. Stability diagram of axisymmetric liquid bridges between coaxial, equal disks, without solid body rotation ( $e=h=B_{l}=W e=0, B_{a} \neq 0$ ). Numbers on the curves indicate the value of the axial Bond number $B_{a}$.

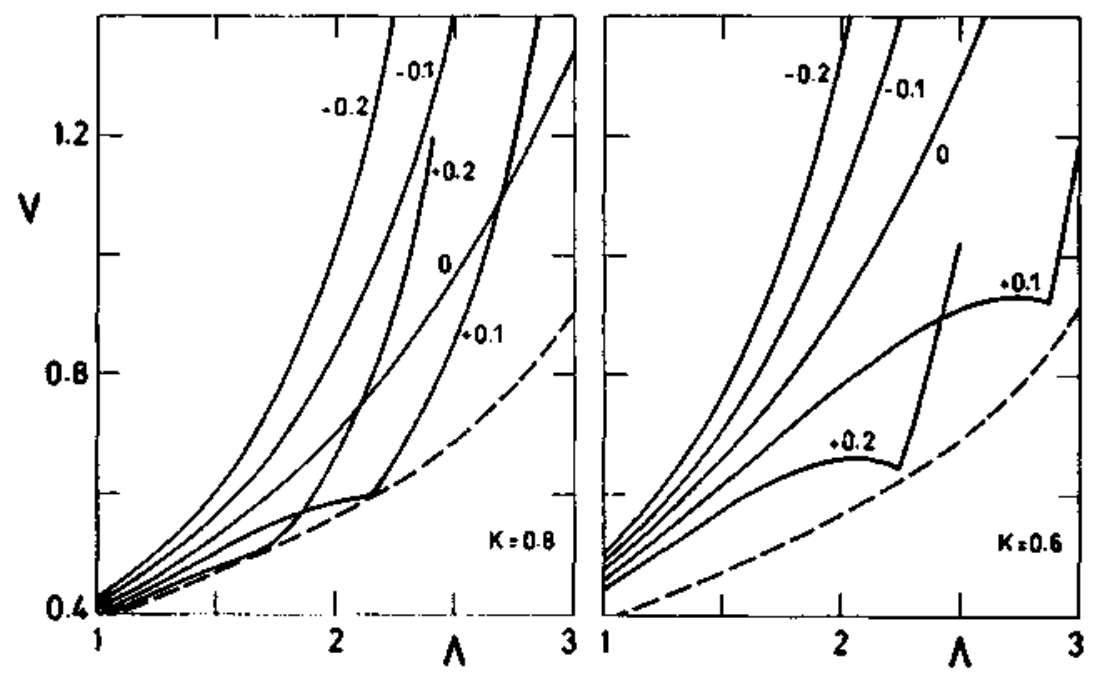

Fig. 5. Stability diagrams of axisymmetric liquid bridge between coaxial, unequal disks, without solid body rotation $\left(e=B_{l}=W e=0, h \neq 0, B_{a} \neq 0\right)$. Numbers on the curves indicate the value of the axial Bond number $B_{a}$. the dashed line correspond the case $K=1, B_{a}=0$.

\section{SOLID BODY ROTATION}

The case of the stability of a liquid column between equal disks, with cylindrical volume and in gravitationless conditions $\left(h=B_{a}=B_{l}=0, V=1\right.$ ) was first analyzed more than ten years ago. As it is well known the nature of the instability appearing when the liquid bridge rotates as a solid depends on the value of Weber number. According to the linear stability analysis $/ 22$, for low values of the Weber number, $W e \leq 1 / 3$, the loss of stability is due to axisymmetric perturbations (amphora mode), the maximum stable slenderness being $\Lambda_{c r i t}=\pi(1+W e)^{-1 / 2}$. On 
the other hand, if the value of Weber number is high enough, $W e \geq 1 / 3$, the stability loss is due to non-axisymmetric perturbations, and the so-called "C-mode" appears. In this second case the maximum stable slenderness becomes $A_{c r i t}=\pi /(2 W e)$. Some work has been done concerning the stability of liquid bridge having non-cylindrical volume $/ 23 /$, although this problem requires additional efforts.

With respect to the combined effect of both Weber number and axial Bond number, available results demonstrate that within the range of validity of an asymptotic analysis (values of the Weber number close to the critical one and small values of the Bond number) the maximum stable slendemess of an axisymmetric liquid bridge with $h=0$ and $V=1$ becomes

$$
\Lambda_{\text {crit }}=\frac{\pi}{(1+W e)^{1 / 2}}\left[1-\left(\frac{9}{4} \frac{B_{a}}{(1+W e)^{2}}\right)^{2 / 3}\right]
$$

Some numerical results have been published recently /24/ concerning the minimum volume stability limits of rotating axisymmetric liquid bridges. Available results show that solid body rotation decreases the stability region in the $A-V$ plane no matter the liquid bridge configurations are (Figure 6).

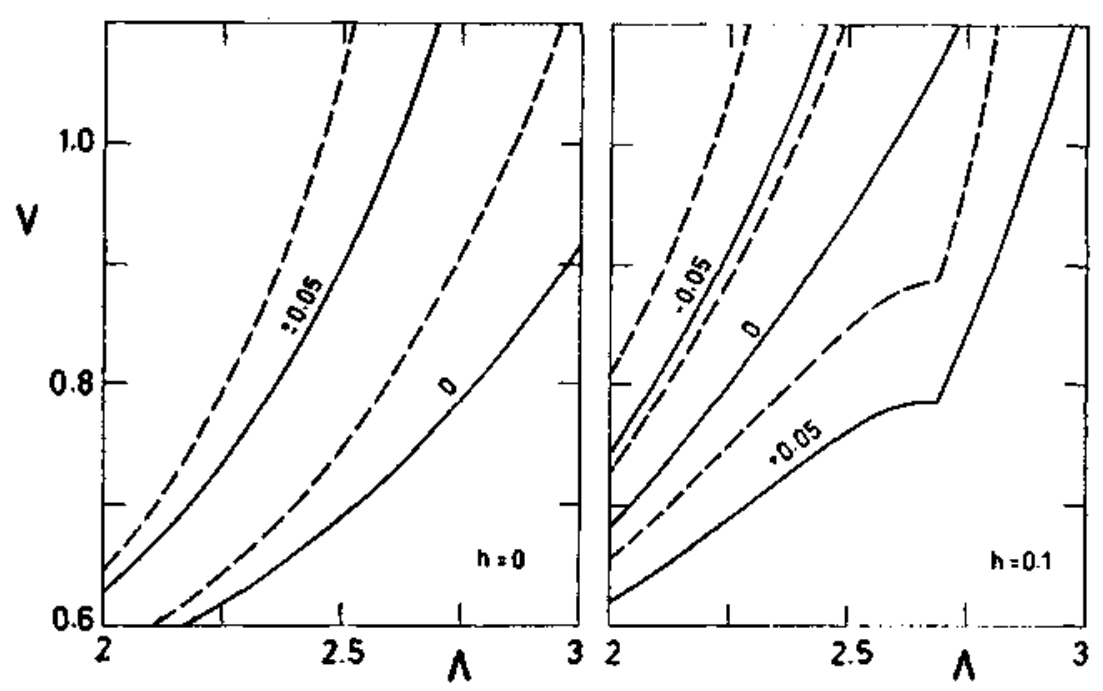

Fig. 6. Stability diagrams of axisymmetric liquid bridges between coaxial disks ( $e=$ $B_{l}=0, B_{a} \neq 0, h \neq 0, W e \neq 0$ ). Numbers on the curves indicate the value of the axial Bond number, $B_{a}$, whereas line type indicates the value of the Weber number: solid lines correspond to $W e=0$ whereas dashed lines correspond to $W e=0.2$.

\section{ECCENTRICITY AND LATERAL BOND NUMBER}

Up to very recently available results related to the effect of non-axisymmetric perturbations like eccentricity or non-axial acceleration were obtained through asymptotic analyses. The maximum stable slenderness of a cylindrical liquid bridge between equal disks subjected to a lateral Bond number $\left(h=e=B_{a}=0, V=1, B_{l} \neq 0\right.$ ) was first analyzed by Coriell, Hardy and Cordes $/ 14 /$. The same problem, but also including the effect of eccentricity was analyzed by Perales $/ 25 /$, although there was a mistake in his conclusions concerning the coupling between eccentricity and lateral Bond number. In a yet unpublished work /26/ the stability limits of liquid bridges with volume close to the cylindrical one and slendernesses close to $\pi$, including both axisymmetric and non-axisymmetric stimuli, have been calculated. According to this study the maximum stable slenderness of an almost cylindrical volume liquid bridge become: 


$$
A_{\text {crit }}=\pi\left[1-\left(\frac{3}{2}\right)^{4 / 3}\left(B_{\mathfrak{a}}-\frac{h}{\pi}-\frac{3}{2 \pi} B_{l} e \cos \beta\right)^{2 / 3}+\frac{1}{2}(V-1)-\frac{\pi^{2}}{4} B_{l}^{2}-\frac{3}{4 \pi^{2}} e^{2}\right]
$$

Obviously, equation (7) is only of application to liquid bridge configurations close to the reference one ( $B_{a}=B_{l}=h=e=0, V=1$ ), but allows us to deduce more general conclusions concerning the influence on the stability limit of the perturbations under consideration. For instance, within this approximation, there is not coupling between the different effects on the variation of the critical slenderness but between $B_{l}$ and $e$ and, when these two effects are considered, another important feature pointed out by equation (7) is that $\Lambda_{c r i}$ does depend on the angle $\beta$ between the plane defined by the axes of the disks and the direction of the lateral component of microgravity. The variation with the eccentricity $e$ and the angle $\beta$ of the parameter $V^{*}=V-1-2(\Lambda / \pi-1)$, which can represent either the minimum stable volume or the maximum stable slenderness, has been plotted in Figure 7 for liquid bridges with $B_{a}=h=0$ and $B_{l}=0.02$. Note that, for fixed $B_{l}$ and $e$,

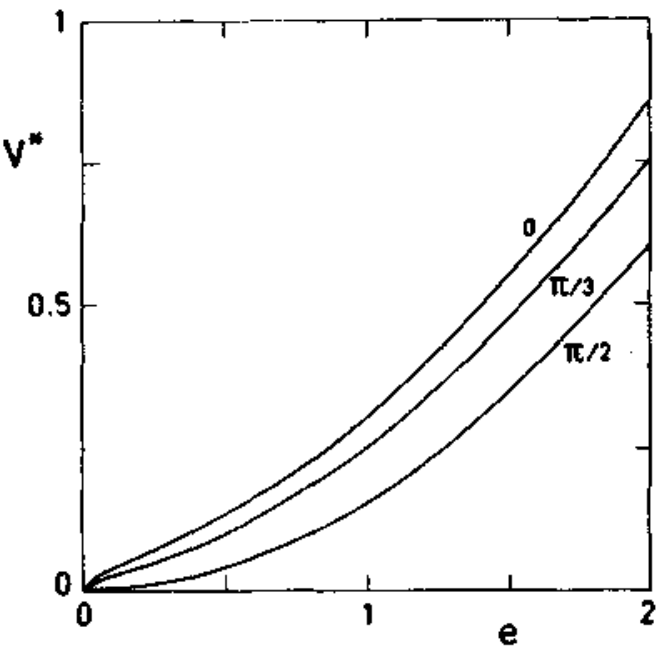

Fig. 7. Minimum reduced volume $V^{*}$ of liquid bridges between non-coaxial, equal disks without solid body rotation in a lateral gravity field $\left(h=B_{a}\right.$ $=W e=0, e \neq 0, B_{l}=0.02$ ). Numbers on the curves indicate the value of the angle $\beta$ between lateral Bond number and the plane defined by the axes of the disks. the stability limit can dramatically change depending on the angle $\beta$.

Another important characteristic of the stability of liquid bridges that must be remarked is that the combined effect of both lateral Bond number and eccentricity (the term in $B e$ ) can be a stabilizing factor for the liquid column. Observe that, leaving apart the combined effect of axial Bond number and unequal disks, in the case of non-coaxial disks the liquid bridge can be more stable if the acceleration has both axial and lateral components than if only one of them is acting on the liquid bridge.

The influence on the minimum volume stability limits of either lateral Bond number or eccentricity has been very recently analyzed numerically by Laverón $27 \%$. The variation with the lateral Bond number of the minimum volume stability limits of liquid bridges between equal disks and without eccentricity is shown in Figure 8 ( $h=e=B_{a}=W e=0$, $B_{l} \neq 0$ ), whereas the combined influence of both lateral and axial Bond numbers is shown in Figure 9, for case of liquid bridges having cylindrical volume $\left(h=e=W e=0, V=1, B_{a} \neq 0, B_{l}\right.$ $\neq 0$ ). Finally, the dependence of the maximum stable slenderness on the eccentricity of cylindrical volume liquid bridges $\left(h=B_{a}=B_{l}=W e=0, V=1, e \neq 0\right.$ ) is shown in Figure 10. In this plot the solid line corresponds to numerical results whereas the dashed line indicates the results given by the asymptotic expression (7). No results have been published concerning the stability limits of maximum volume of liquid bridges subjected to non-axisymmetric stimuli like eccentricity or lateral Bond number. 


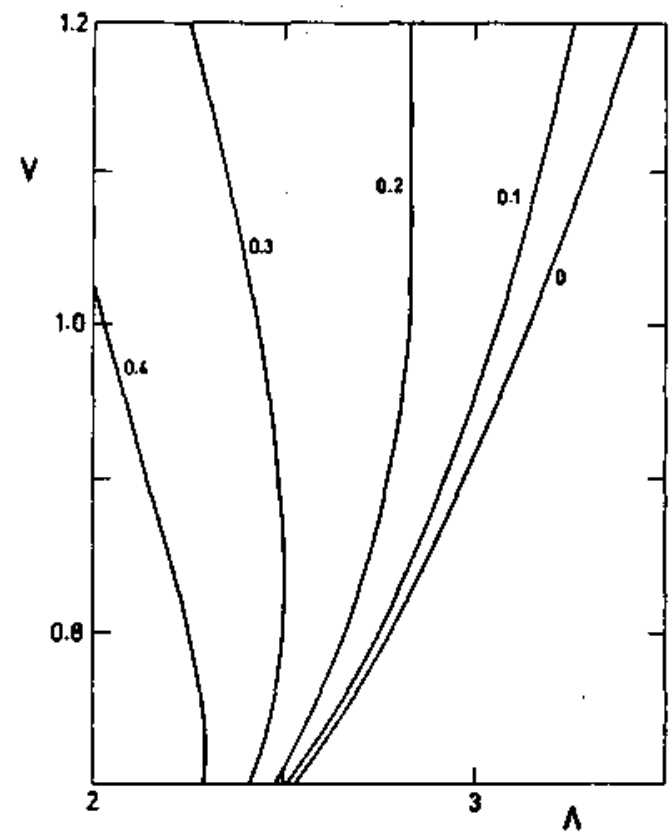

Fig. 8. Stability diagram of non-axisymmetric liquid bridges between coaxial, equal disks without solid body rotation $\left(e=h=B_{a}=W e=0, B_{l} \neq 0\right.$ ). Numbers on the curves indicate the value of the lateral Bond number $B_{l}$.

\section{CONCLUSIONS}

In this paper a brief review on the stability limits of liquid bridges has been presented. Stability diagrams in the $\Lambda-V$ plane have been presented for a wide variety of stimuli, either axisymmetric, or non-axisymmetric. There are other stimuli whose effects on stability limits have been analyzed, mainly the influence of electric fields $128,29,30 /$, but these are out of the scope of this review.

It can be concluded that the stability of liquid bridges has paid the attention of numerous scientists in the last years, and because of that the knowledge on this fluid physics problem has been increased by orders of magnitude during the last decade. However, these are still some aspects of the hydrostatic of liquid bridges which require additional effort: mainly those related to the maximum volume stability limits of liquid bridges subjected to nonaxisymmetric perturbations.

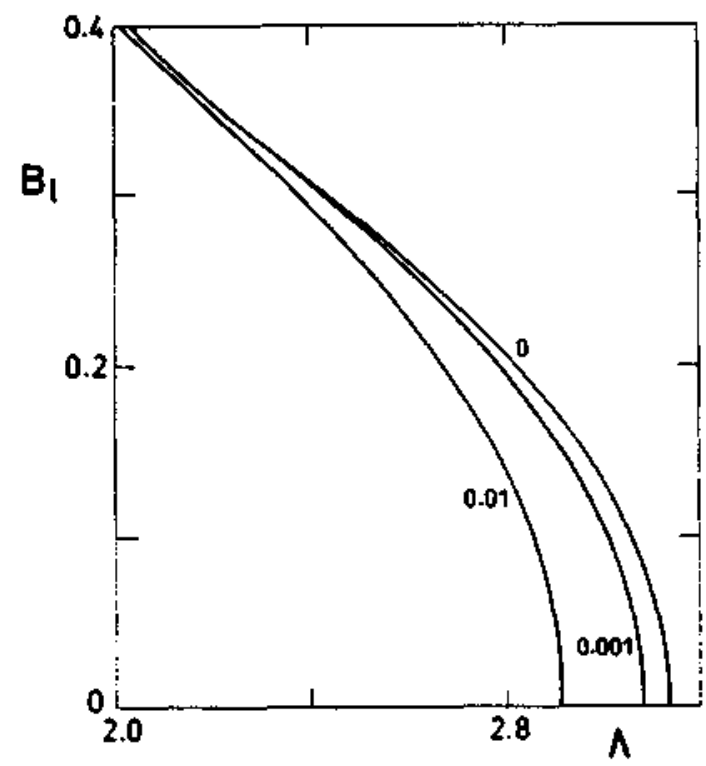

Fig. 9. Maximum stable slenderness of liquid bridges between coaxial, equal disks, without solid body rotation and having cylindrical volume $(V=1$, $e=h=W e=0, B_{a} \neq 0, B_{l} \neq 0$ ). Numbers on the curves indicate the value of the axial Bond number $B_{a}$. 


\section{ACKNOWLEDGEMENTS}

This work has been supported by the Spanish Comisión Interministerial de Ciencia y Tecnología (CICYT Project ESP 92-0001-CP).

\section{REFERENCES}

1. R.D. Gillette and R.C. Dyson, Stability of fluid interfaces of revolution between equal solid circular plates, Chem. Eng. J., 2. 44-54 (1971).

2. I. Da Riva and I. Martínez, Floating zone stability (Exp. 1-ES-331), ESA $S P-142,67-73$ (1979).

3. M.A. Erle, R.D. Gillette and D.C. Dyson, Stability of interfaces of revolution with constant surface tension. The case of the catenoid, Chem. Eng. J., $\perp$, 97-109 (1970).

4. L.A. Slobozhanin, Problems on the stability of liquids in equilibrium, appearing in spatial technology, in Hydromechanics and Heat and Mass Transfer in Zero-Gravity, 9-24 (in Russian), Nauka, Moscow (1982).

5. I. Martínez, Floating zone. Equilibrium shapes and stability criteria, in COSPAR Space Research XVIII, 519-522, Pergamon Press (1978).

6. E.A. Boucher and T.G. Jones, Equilibrium and stability characteristics of zero-gravity fluid bridges constrained between equal solid rods, J. Colloid Interface Sci., 126, 469-481 (1988).

7. G.C. Mason, An experimental determination of the stable length of cylindrical liquid bubbles, J. Colloid Interface Sci., 32, 172-176 (1970).

8. J.R. Carruthers and M. Grasso, Studies of floating liquid zones in simulated zero gravity, $J$. Appl. Phys., 43, 436-445 (1972).

9. A. Sanz and J. Martínez, Minimum volume for a liquid bridge between equal disks, $J$. Colloid Interface Sci., 23, 235-240 ( 1983).

10. I. Martínez, Stability of axisymmetric liquid bridges, ESA SP-191, 267-273 (1983).

11. I. Martinez and J.M. Perales, Liquid bridge stability data, $J$. Crystal Growth, 78, 369-378 (1986).

12. J.M. Perales, private communication (1994).

13. S.R. Coriell, S.C. Hardy and M.R. Cordes, Stability of liquid zones, J. Colloid Interface Sci., 60, 126-136 (1977). 
14. S.R. Coriell and M.R. Cordes, Theory of molten shape and stability, J. Crystal Growth, 42 . 466-472 (1977).

15. E.A. Boucher and M.J.B. Evans, Properties of fluid bridges between solids in a gravitational field, J. Colloid Interface Sci., 75, 409-418 (1980).

16. I. Martínez, J.M. Haynes and D. Langbein, Fluid sciences and materials sciences in space, Springer-Verlag (1987).

17. I Meseguer and A. Sanz, Numerical and experimental study of the dynamics of axisymmetric liquid bridges, J. Fluid Mech., 153, 83-101 (1985).

18. N.A. Bezdenejnykh, J. Meseguer and J.M. Perales, Experimental analysis of stability limits of capillary liquid bridges, Phys. Fluids $A, \underline{4}, 677-680$ (1992).

19. L.A. Slobozhanin and J.M. Perales, Stability of liquid bridges between equal disks in an axial gravity field, Phys. Fluids A, 5, 1305-1314 (1993).

20. J. Meseguer, Stability of slender, axisymmetric liquid bridges between unequal disks, $J$. Crystal Growth, 67, 141-143 (1984).

21. J.M. Perales, J. Meseguer and I. Martínez, Minimum volume stability limits for axisymmetric liquid bridges subject to steady axial acceleration, J. Crystal Growth, 110 855-861 (1991).

22. J.M. Vega and J.M. Perales, Almost cylindrical isorotating liquid bridges for small Bond numbers, ESA $S P-191,247-252$ (1983).

23. R.A. Brown and L.E. Scriven, The shapes and stability of captive rotating drops, Phil. Trans. R. Soc. Lond. A, 297, 51-79 (1980).

24. I. Martínez, J.M. Perales and M. Gómez, Effects of axial and centrifugal forces on the stability of liquid bridges, ESA SP-333, 123-130 (1992).

25. J.M. Perales, Non-axisymmetric effects on long liquid bridges, Acta Astronautica, 15, 561-565 (1987).

26. J. Meseguer, N.A. Bezdenejnykh, J.M. Perales and P. Rodríguez de Francisco, Theoretical and experimental analysis of stability linits of non-axisymmetric liquid bridges, submitted to Microgravity Science and Technology (1994).

27. A. Laverón, Estabilidad de puentes líquidos no axilsimétricos. Tesis Doctoral, (in Spanish), Universidad Politécnica de Madrid (1994)

28. H. Gonzalez, F.M.J. Mc Cluskey, A. Castellanos and A. Barrero, Stabilization of dielectric liquid bridges by electric fields in the absence of gravity, J. Fluid Mech., 206, 545-561 (1989).

29. H. González and A. Castellanos, The effect of residual gravity on the stability liquid columns subjected to electric fields, J. Fluid Mech., 249, 185-206 (1993).

30. A. Ramos and A. Castellanos, Bifurcation diagrams of axisymmetric liquid bridges of arbitrary volume in electric and gravitational fields, J. Fluid Mech., 249, 207-225 (1993). 